\title{
Quantum tunneling of domain walls in ferromagnets
}

\author{
E. M. Chudnovsky \\ Physics Department, Lehman College, The City University of New York, Bedford Park Boulevard West, Bronx, New York 10468 \\ O. Iglesias \\ Departamento Física Fonamental, Facultat de Física, Universitat de Barcelona, Diagonal 647, 08028 Barcelona, Spain \\ P. C. E. Stamp \\ Physics Department, University of British Columbia, 6224 Agricultural Road, Vancouver, British Columbia, Canada V6T $1 Z 1$
}

(Received 12 December 1991; revised manuscript received 19 March 1992)

\begin{abstract}
We present a theoretical study of the quantum depinning of domain walls. Our approach extends earlier work by Stamp and confirms his suggestion that quantum tunneling of domain walls in ferromagnets may reveal itself at a macroscopic level in a manner similar to the Josephson effect in superconductors. The rate of tunneling of a domain wall through a barrier formed by a planar defect is calculated in terms of macroscopic parameters of the ferromagnet. A universal behavior of the WKB exponent in the iimit of small barriers is demonstrated. The effect of dissipation on the tunneling rate is studied. It is argued that quantum diffusion of domain walls apparently explains a nonthermal magnetic relaxation observed in some materials at low temperatures.
\end{abstract}

\section{INTRODUCTION}

The physics of magnetism of solids consists of two major domains: quantum mechanical and classical. The quantum-mechanical domain is conventionally concerned with the nature of the elementary carriers of magnetism (localized moments, itinerant electrons, etc.) and adequate models of their interactions. The classical domain is designed to deal with experimental data on the coherent behavior of a large number of elementary magnetic moments. It operates with a continuous magnetization of a solid, $\mathbf{M}(\mathbf{x}, t)$, and phenomenological constants (exchange, magnetic anisotropy, etc.). This approach, traditionally called micromagnetism, has a long record of successful applications to ferromagnetic resonance, monodomain particles, spin waves, domain walls, and other magnetic objects whose size ranges from a few angströms to a macroscopic scale.

Recently, it has been realized that the field $\mathbf{M}(\mathbf{x}, t)$ may display fascinating quantum behavior independently of the underlying spin structure of the magnetization. In particular, it may tunnel from a metastable configuration to a more stable magnetic state. A general approach to the tunneling of $\mathbf{M}(\mathbf{x})$ has been formulated in Ref. 1 . It is based upon the observation that the equations of micromagnetic theory may be obtained as extremal trajectories of a some uniquely defined magnetic action,

$$
S=\int d t \int d^{3} x \mathcal{L}[\mathbf{M}(\mathbf{x}, t)]
$$

$\mathcal{L}$ being the Lagrangian density. The tunneling rate is then given by the path integral

$$
\int D\{\mathbf{M}(\mathbf{x}, t)\} \exp \left[\frac{i}{\hbar} S\right),
$$

over all $\mathbf{M}(\mathbf{x}, t)$ trajectories leading from the initial configuration $\mathbf{M}_{1}(\mathbf{x})$ to the final configuration $\mathbf{M}_{2}(\mathbf{x})$. The most attractive feature of this approach is that the WKB exponent of the tunneling is given by the imaginary-time extremal solution (instanton) of the wellknown classical equation for $\mathbf{M}(\mathbf{x}, t)$, which, in some cases, can be obtained exactly. Examples are tunneling of the magnetic moment between equilibrium orientations in a monodomain particle, ${ }^{2}$ quantum nucleation of the magnetization reversal nucleus in a bulk ferromagnet, ${ }^{3}$ and tunneling of domain walls, ${ }^{4}$ the main subject of this paper.

One may feel uneasy about applying quantum mechanics to a classical variable

$$
\mathbf{M}(\mathbf{x}, t)=\left\langle 2 \mu_{B} \sum_{i} \hat{s}_{i} \delta\left(\mathbf{x}-\mathbf{x}_{i}\right)\right\rangle .
$$

Note in this connection that the problem is not essentially different from the $\alpha$ decay of a nucleus. For the latter problem one ignores the fact that the $\alpha$ particle is formed by four operators of nucleons (or 12 quark operators), which is justified by the strong coupling of nucleons inside the $\alpha$ particle. Similar to that problem, the strong exchange coupling of spins in a ferromagnet allows one to consider $\mathbf{M}(\mathbf{x}, t)$, not individual spins, ${ }^{1}$ as a tunneling variable. The Feynman approach to quantum field theory, Eq. (2), does not specify how fundamental the field should be. It allows one to develop quantum theory based upon a classical Lagrangian only. There are two questions, however, which one should be concerned about when applying quantum mechanics to a macroscopic variable. The first is the magnitude of the imaginary-time action associated with the quantum process. It should be sufficiently small, say not to exceed $30 \hbar$; otherwise quantum effects will be exponentially suppressed, as they usually are in the macroscopic world. 
For the tunneling of a macroscopic variable the value of the WKB exponent depends on the height and the width of the energy barrier which should be, therefore, made as small as possible, to observe the effect. Magnetic systems provide a remarkable opportunity for that. Indeed, the presence of various energy barriers and metastable states in ferromagnets is manifested by their pronounced hysteresis phenomena. This metastability may be diminished and eventually removed by increasing the magnetic field. Making any particular barrier as small as possible is, therefore, a question of the accuracy with which one can control the magnetic field. It has been shown ${ }^{2-4}$ that the condition of a small barrier can be easily satisfied, making possible a coherent tunneling of a large number of spins.

The second reason of concern comes from the coupling of the tunneling variable, $M(x, t)$, with other degrees of freedom, e.g., phonons, photons, etc. Since the limit of small barrier is of interest, it is not clear beforehand how weak the coupling should be, compared with other energies in the problem, in order not to undermine the tunneling. An answer to this question was given for couplings to the environment which are linear in the environmental variables by Caldeira and Leggett. ${ }^{5}$ They found that most serious effects come from Ohmic coupling, in which the classical equations of motion, has the form

$$
\ddot{x}+v \dot{x}=-\frac{1}{m} \frac{\partial U}{\partial x}
$$

for small displacements of the tunneling variable $x ; v$ being the constant of Ohmic dissipation. For such a linear coupling to the environment, the remarkable result of Caldeira and Leggett is that the effect of the environment on the WKB exponent, $B$, can be expressed in terms of the constant $v$,

$$
B \sim B_{0}\left(1+\frac{v}{\omega_{0}}\right),
$$

where $B_{0} \sim U_{0} / \hbar \omega_{0} \gg 1$ is the WKB exponent in the absence of dissipation, $U_{0}$ is the height of the barrier, $\omega_{0}$ is a characteristic instanton frequency. Quantum mechanics gives $\omega_{0} \propto \sqrt{U_{0} / m}$. Correspondingly, the effect of dissipation becomes significant in the limit of small $U_{0}$. The question of whether dissipation is important then becomes equivalent to the following two questions: (i) are there any processes yielding Ohmic dissipation and if so (ii) what is the dissipation coefficient $v$ ?

Tunneling exists in the presence of metastable states. There are two major sources of metastability in ferromagnets. The first comes from the fundamental nonlinearity of the magnetic energy. It leads to the existence of several directions of easy magnetization and to the appearance of magnetic domains. The second source of metastability are various kinds of crystal defect. Due to such defects the energy of a domain wall fluctuates over the volume of the sample. This leads to the pinning of walls by defects. In the presence of a magnetic field, domains whose magnetization is aligned with the field tend to grow, while domains whose magnetization is opposite to the field tend to collapse. The force on the domain wall is proportional to the field. As the field grows, this force eventually exceeds the pinning force and the wall becomes mobile. However, even well below the coercive field, one usually observes some slow dynamics of the magnetization due to the thermal overbarrier diffusion of domain walls in the pinning potential. The rate of the thermal transition through the pinning barrier is governed by the Boltzmann exponent, $\exp \left(-U_{0} / k_{B} T\right)$. These transitions die out as the temperature goes to zero. In this paper we will study processes of quantum tunneling of domain walls. The probability of the tunneling is governed by the temperature independent WKB exponent, $\exp (-B)$. Two conditions must be satisfied to make quantum effects observable: First, the rate of quantum tunneling must be significant, which requires a small barrier. Second, comparing thermal and quantum exponents, one finds that quantum transitions dominate at $k_{B} T<k_{B} T_{c}=U_{0} / B$. Consequently, while being concerned about a small barrier, one should also take care that the corresponding crossover temperature, $T_{c}$, is accessible experimentally.

Indirect evidence for "mesoscopic" tunneling of domain walls (involving $\sim 10^{2}-10^{3}$ spins) has been around for some time. Egami ${ }^{6}$ suggested that the temperature-independent magnetic aftereffects observed at low temperature by Barbara et $a l .{ }^{7}$ (see also Ref. 8), in highly anisotropic magnets, might be due to quantum diffusion of domain walls. However his ideas only apply to extremely narrow walls which have the width of the order of the lattice spacing and are pinned by the crystal potential itself. This implies very large values of magnetic anisotropy. Typical walls have a thickness of a few hundred angströms and are pinned by defects. A simple WKB approximation for the tunneling of domain walls through small obstacles was suggested by a number of authors ${ }^{9}$ but no attempt was made to calculate the tunneling rate in the spirit of the Caldeira-Leggett approach. Recently, however, Stamp ${ }^{4}$ showed how to do this for a planar wall coupled to a point defect. The tunneling rate and contributions of various dissipative interactions were estimated in terms of the coercive field, exchange, and anisotropy constants. Most importantly, it was shown that the tunneling of a domain wall involving a macroscopically large number of spins may occur. Recent experiments seem to provide more evidence for such tunneling, at least on the mesoscopic scale. ${ }^{10-12}$

Quantum diffusion of a domain wall occurs via discrete changes of its local geometry due to the tunneling of the wall between metastable minima of the pinning potential. One of the goals of this paper is to develop a formulation of the tunneling problem, which takes the curvature effects into consideration. The domain wall corresponds to the rotation of the vector of magnetization from one magnetic domain to another. Tunneling of the wall is, thus, a transition between two $M(x)$ configurations, which may be treated in terms of Eq. (2). For the wall tunneling through the pinning barrier, however, it is hopeless to find the corresponding imaginary-time solution of Eq. (6). Instead, we will treat the domain wall as a two-dimensional object characterized by the metric $g_{i k}$ or by the equation of the surface $Z(x, y, t)$. The tunneling of the wall then corresponds to the instanton connecting the 
initial and the final geometries of the surface. In this approach the dependence of the width and the energy of the wall on its velocity and orientation will be left out of the picture. We will demonstrate, however, that our approximation is rather good in the limit of a small barrier, which is of primary interest for the tunneling problem.

The effect of the environment on the domain-wall tunneling includes interaction with itinerant electrons, magnons, phonons, and the electromagnetic field. The model for dissipation due to magnetoelastic interactions was formulated in Ref. 2 and worked out in detail for onephonon processes by Garg and Kim. ${ }^{13}$ This interaction is non-Ohmic and its relative contribution to the tunneling rate turns out to be very small, typically of the order of $10^{-6}$. We will show that only Ohmic couplings may have a noticeable effect on the tunneling rate and suggest an estimation of this effect in terms of the mobility of the domain wall. We will see that the most important process in metals is the interaction with conduction electrons. However, in insulators the interaction with magnons is the main source of dissipation. These two processes must be handled separately.

The dissipation of the domain-wall motion in metals is due to eddy currents, that is, Ohmic in nature. It should be sufficient, therefore, to describe the magnetization dynamics in metals in terms of the Landau-Lifshits-Gilbert equation:

$$
\frac{\partial \mathbf{M}}{\partial t}=-\gamma \mathbf{M} \times \frac{\delta E}{\delta \mathbf{M}}-\frac{v}{M^{2}} \mathbf{M} \times\left(\mathbf{M} \times \frac{\delta E}{\delta \mathbf{M}}\right),
$$

where $\mathbf{M}^{2}=M_{0}^{2}=$ const, $E(\mathbf{M})$ is the appropriate energy functional, $\gamma$ is the gyromagnetic ratio, and $v$ is the dissipation constant responsible for the finite width of the ferromagnetic resonance. We will investigate this question in more detail.

There are two reasons why Eq. (6) cannot be applied to dissipation problems involving magnons. First, processes involving magnons do not conserve $|\mathbf{M}|$. Second, interaction of the magnetization vector with magnons is nonlinear in nature. The way to map this nonlinear interaction onto Caldeira-Leggett coupling has been pointed out in Ref. 4. In this paper we will give some details of these calculations, and will extend the analysis of magnon dissipation for planar walls ${ }^{4}$ to the case of weakly curved walls.

Our main purpose will be the calculation of the tunneling rate, $P=A \exp (-B)$, and the crossover temperature, $T_{c}=U_{0} / B$, in terms of macroscopic parameters of the system, and the effects of dissipation on these quantities. The model is formulated in Sec. II. In Sec. III we calculate the WKB exponent for tunneling through a planar defect. Two cases are studied: tunneling of the flat portion of the wall through a small defect and tunneling via quantum nucleation through a larger defect. The crossover temperature is obtained in Sec. IV. In Sec. V we compute the preexponential factor $A$. In Sec. VI different mechanisms of dissipation and their contribution to the tunneling rate are studied. Results and their relevance to recent experimental data are discussed in Sec. VII.

\section{THE MODEL}

We will consider the domain wall as a surface (Fig. 1) of energy $\sigma_{0}$, per unit area. In general the energy density $\sigma(\mathbf{P}, \mathbf{R})$ is a functional of generalized coordinates $\mathbf{P}(\xi)$ (the momentum density) and $\mathbf{R}(\xi)$ (the radius vector of the wall); here $\xi=\left(\xi_{0}, \xi_{1}, \xi_{2}\right)$ parametrizes the wall surface, with $\xi_{0}$ representing time, and $\left(\xi_{1}, \xi_{2}\right)$, the spatial coordinates on the wall. Now in general the dynamics of the wall is governed by the Slonczewski equations, ${ }^{14}$ which take the form

$$
\frac{\delta \sigma}{\delta \mathbf{R}(\xi)}=-\dot{\mathbf{P}}(\xi), \quad \frac{\delta \sigma}{\delta \mathbf{P}(\xi)}=\dot{\mathbf{R}}(\xi) .
$$

For an arbitrary wall these can be very complicated, but for the gently curved wall of Fig. 1 one has a wall energy density $\sigma(\mathbf{P}, \mathbf{R})$ of the form

$$
\begin{gathered}
\sigma=\sigma_{0}\left[\left[1+\frac{\gamma^{2} \delta^{2}}{4 M_{0}^{2}}\left(\nabla P_{\perp}\right)^{2}\right.\right. \\
\left.\left.\quad+\frac{\dot{Z}^{2}}{v_{0}^{2}\left[1+(\nabla Z)^{2}\right]}\right]\left[1+(\nabla Z)^{2}\right]\right]^{1 / 2},
\end{gathered}
$$

where $\mathbf{P}_{1}(x, y, t)$ is the component of wall momentum density perpendicular to the wall surface $Z(x, y, t), \delta$ is the wall thickness, $v_{0}$ is the limiting (Walker) velocity of the wall, and $\sigma_{0}$ is the constant energy density of a planar stationary wall.

The term proportional to $\left(\nabla \mathbf{P}_{\perp}\right)^{2}$ in Eq. (8) corresponds to an inhomogeneous exchange energy in the wall. It has the order of $\delta^{2} / R_{0}^{2}$ compared to the term $\dot{Z}^{2} / v_{0}^{2} ; R_{0}$ being the curvature radius of the wall. The main approximation we shall make in this paper is to assume weak curvature $\left(R_{0} \gg \delta\right)$ and slow $\left(|\dot{Z}|<<v_{0}\right)$ motion of the wall. We will see that these conditions are always satisfied for the tunneling problem in the limit of small barrier. The equations of motion (7) then become equivalent to the covariant action,

$$
S_{0}=-\sigma_{0} \int d^{3} \xi \sqrt{g(\xi)}
$$

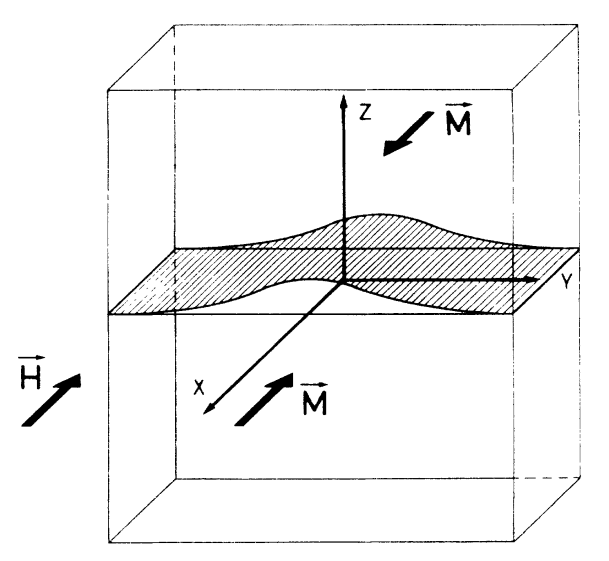

FIG. 1. Decoupling of the domain wall from a planar defect located in the $X Y$ plane. 
where $g(\xi)=\operatorname{det}\left[g_{i j}(\xi)\right], g_{i j}$ is the metric tensor,

$$
g_{i j}=\frac{\partial \mathbf{R}(\xi)}{\partial \xi_{i}} \cdot \frac{\partial \mathbf{R}(\xi)}{\partial \xi_{j}} \text {. }
$$

To Eq. (9) we should also add the terms coming from a pinning potential $U[\mathbf{R}(\xi)]$ and a volume magnetic energy coming from the magnetic field $\mathbf{H}$; this gives a further contribution $S_{p}+S_{m}$ to the action, where

$$
\begin{aligned}
S_{p}+S_{m}= & -\int d^{3} \xi \sqrt{g(\xi)} U[\mathbf{R}(\xi)] \\
& -\int d t \int d^{3} R \mathbf{M}(\mathbf{R}) \cdot \mathbf{H} .
\end{aligned}
$$

It is convenient now to convert to the parametrization $\xi_{0}=t, \xi_{1}=x, \xi_{2}=y$, and describe the wall by the singlevalued function $Z(x, y, t)$ [note that for cylindrical magnetic-bubble nucleation ${ }^{3}$ this would not be convenient; a better choice would be $\boldsymbol{Z}=\boldsymbol{Z}(\rho, \phi, t)$, with $z, \rho, \phi$ being cylindrical coordinates]. With the approximations described above we can then rewrite Eq. (9) as

$$
S_{0}=-\sigma_{0} \int d t d x d y\left[1+(\nabla Z)^{2}-\frac{1}{v_{0}^{2}} \dot{Z}^{2}\right]^{1 / 2} \text {. }
$$

The physical meaning of Eq. (12) becomes obvious in the limit of the small velocity of the wall, $|\dot{Z}|<<v_{0}$. Expanding the square root under the integral, one obtains

$$
S_{0}=\int d t \int \frac{d x d y}{\cos \theta} \frac{m_{0} v_{1}^{2}}{2}-\int d t \int \frac{d x d y}{\cos \theta} \sigma_{0},
$$

where $\theta(x, y, t)$ is the angle that the vector normal to the surface makes with the $Z$ axis, $v_{\perp}(x, y, t)=\dot{Z} \cos \theta$ is the local velocity of the wall, $m_{0}=\sigma_{0} / v_{0}^{2}$ is the mass of the unit area of the wall. Here $d x d y / \cos \theta$ is the element of the surface, $\cos \theta=\left[1+\left(\partial_{x} Z\right)^{2}+\left(\partial_{y} Z\right)^{2}\right]^{-1 / 2}$. Correspondingly the two terms in Eq. (13) represent the kinetic and the surface energy contributions to the action of the wall.

It is convenient to consider the imaginary time $\tau=i t$ and to use dimensionless variables,

$$
\begin{aligned}
& x_{0}=\omega \tau, \quad x_{1}=x / \delta, \\
& x_{2}=y / \delta, \quad \bar{z}=Z / \delta, \\
& u=U(x, y, z) / \sigma_{0}, \quad h=2 M_{0} H \delta / \sigma_{0},
\end{aligned}
$$

where $\delta$ is the domain wall thickness, $\omega=v_{0} / \delta$. Then the total Euclidean action, $S_{E}=i S$, becomes

$$
\begin{aligned}
S_{E}=-\frac{\sigma_{0} \delta^{2}}{\omega} \int d^{3} x & \left\{\left(1+[\nabla \bar{z}(\mathbf{x})]^{2}\right)^{1 / 2}\right. \\
& \left.+u\left(x_{1}, x_{2}, \bar{z}\right)-h \bar{z}(\mathbf{x})\right\},
\end{aligned}
$$

where $\mathrm{x}=\left(x_{0}, x_{1}, x_{2}\right), \nabla=\left(\partial_{0}, \partial_{1}, \partial_{2}\right)$.

The parameters of micromagnetic theory, which represent macroscopic properties of ferromagnets, are exchange, $A_{e}(\mathrm{erg} / \mathrm{cm})$, and anisotropy, $K\left(\mathrm{erg} / \mathrm{cm}^{3}\right)$, constants. It may be helpful to have the dependence of our parameters $\sigma_{0}, \delta, \omega$ on these constants. We will illustrate it by the example of a rhombic crystal with the magnetic anisotropy energy characterized by two positive constants, $K_{\|}$and $K_{1}$ :

$$
E_{\alpha}=-K_{\|} \frac{M_{x}^{2}}{M_{0_{2}}}+K_{\perp} \frac{M_{z}^{2}}{M_{0}^{2}} .
$$

This describes a magnet having the $X$ axis as the easy magnetization direction, and the $X Y$ plane as the easy plane. Consequently, the magnetization inside the domain wall rotates in the XY plane (see Fig. 1). For such a wall

$$
\sigma_{0}=4\left(A_{e} K_{\|}\right)^{1 / 2}, \quad \delta=\left(A_{e} / K_{\|}\right)^{1 / 2} .
$$

The limiting velocity is given by (see, e.g., Ref. 14)

$$
v_{0}=\frac{2 \gamma}{M_{0}}\left(A_{e} K_{\|}\right)^{1 / 2}\left[\left(1+\frac{K_{\perp}}{K_{\|}}\right)^{1 / 2}-1\right] .
$$

Note, that in the limit of an uniaxial crystal $\left(K_{1}=0\right)$ the wall has an infinite mass, $m_{0}=\sigma_{0} / v_{0}^{2}$, and, therefore, cannot move or tunnel. This is due to the fact that the Hamiltonian, $-K_{\|} \hat{s}_{x}^{2}$, produced in this limit by Eq. (16), commutes with the spin operator $\widehat{s}_{x}$, so that $s_{x}$ on each side of the wall becomes a conserved quantum number. In fact, however, the magnetic dipole interaction, which is not represented in Eq. (16), violates the commutation of the Hamitlonian with $\hat{s}_{x}$ even for an uniaxial crystal, leading to the replacement of $K_{1}$ by $2 \pi M_{0}^{2}$. The frequency $\omega$ depends on anisotropy constants, but not on the exchange,

$$
\omega=\gamma H_{a}\left[\left(1+\frac{K_{\perp}}{K_{\|}}\right]^{1 / 2}-1\right],
$$

where we have introduced the anisotropy field $H_{a}=2 K_{\|} / M_{0}$. As we will see below, $\hbar \omega / k_{B}$ determines the scale of the crossover temperature $T_{c}$.

Although we have not specified the pinning potential $U$, some important conclusions can be given on the magnitude of the reduced potential $u=U / \sigma_{0}$ in Eq. (15). The surface energy of the wall depends on the strength of the local exchange and anisotropy, Eq. (17). Fluctuations in $A_{e}$ and $K_{\|}$due to defects, and resulting fluctuations in $\sigma_{0}$, are responsible for the pinning. The wall is attracted to regions where $\sigma_{0}$ is less than it is in an ideal crystal. Correspondingly, the value of $\sigma_{0}$ in an ideal crystal is the energy available for pinning. This suggests that the reduced pinning potential, $u=U / \sigma_{0}$, satisfies $u<1$. A similar argument can be made about the reduced field $h$. From Eq. (17) we get $h=H / H_{a}$. Energy barriers in ferromagnets normally exist at $H<H_{c} \leq H_{a}$, which gives $h \leq 1$.

Equation (15) allows one to compute the WKB exponent for an arbitrary curvature of the wall and an arbitrary pinning potential. We will concentrate on the case of small curvature and "slow" instantons, $(\nabla \bar{z})^{2}<<1$, whose significance will be explained below. To simplify the problem we will also study planar defects only, for which $u=u(z)$. The latter may be justified by the fact that, because of energy considerations walls tend to match with planar defects or with groups of defects con- 
centrated in one plane. One can also imagine a specific experiment where the wall tunnels through a planar defect. In the limit of small space-time derivatives and $u=u(z), S_{E}$ reduces to

$$
S_{E}=-\frac{\sigma_{0} \delta^{2}}{\omega} \int d^{3} x\left[\frac{1}{2}(\nabla \bar{z})^{2}+u(\bar{z})-h \bar{z}\right] .
$$

\section{THE WKB EXPONENT}

\section{A. Tunneling in $1+1$ dimensions}

Consider a flat domain wall of a small area $A_{w}$, tunneling through a planar defect of the same area. This may be treated as an approximation for the tunneling through a small defect, or may correspond to the situation when the wall is coupled to a planar defect within a very thin wire, with $A_{w}$ being the cross section of the wire. Then Eq. (20) becomes

$S_{E}=-\frac{\sigma_{0} A_{w}}{\omega} \int d x_{0}\left[\frac{1}{2}\left[\frac{d \bar{z}}{d x_{0}}\right]^{2}+u(\bar{z})-h \bar{z}\right]$.

The extremal trajectory satisfies

$$
\frac{d^{2} \bar{z}}{d x_{0}^{2}}=\frac{d u}{d \bar{z}}-h
$$

The total potential, $u(\bar{z})-h \bar{z}$, of the general form is shown in Fig. 2(a). The wall tunnels from $\bar{z}=z_{1}$ to $\bar{z}=z_{2}$. As is shown in Fig. 2(b), $z_{1}$ and $z_{0}$ are solutions of $d u / d \bar{z}=h$. At $h=h_{c}$ the energy barrier disappears. This corresponds to $z_{2} \rightarrow z_{0} \rightarrow z_{1} \rightarrow 0$ at $h \rightarrow h_{c}$. In the case of a small barrier $\left(h \rightarrow h_{c}\right.$ ), the general form of $d u / d \bar{z}$ is [see Fig. 2(b)]

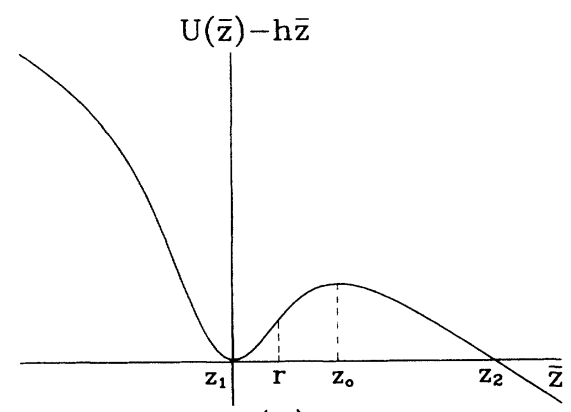

(a)

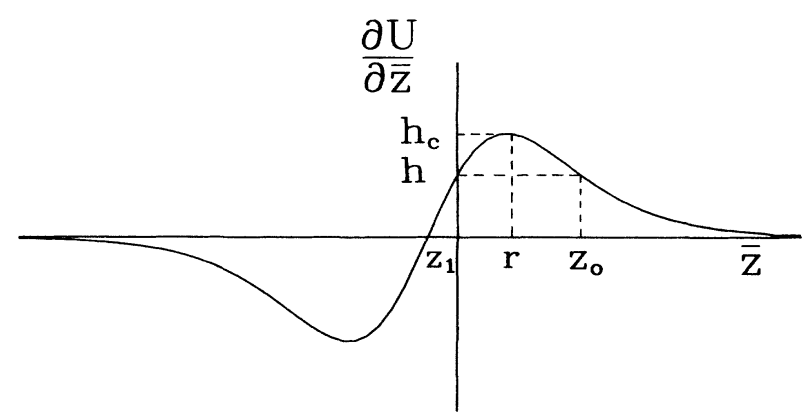

(b)

FIG. 2. (a) The shape of the potential, $u_{0}(z)-h z$. (b) $\partial u_{0} / \partial z$ vs $z$.

$$
\frac{d u}{d \bar{z}}=h_{c}-\frac{1}{2}\left(\frac{\bar{z}-r}{\bar{w}}\right)^{2},
$$

where $\bar{w}$ is the parameter characterizing the shape of the potential, $u(\bar{z})$, produced by the defect. Equation (23) gives

$$
z_{1}=r-\bar{w}\left(2 h_{c} \epsilon\right)^{1 / 2}, \quad z_{0}=r+\bar{w}\left(2 h_{c} \epsilon\right)^{1 / 2},
$$

where we have introduced a small parameter $\epsilon=1-h / h_{c}$. It is convenient to choose the $Z$ axis such that $z_{1}=0$. Then $r=\bar{w}\left(2 h_{c} \epsilon\right)^{1 / 2}, z_{0}=2 \bar{w}\left(2 h_{c} \epsilon\right)^{1 / 2}$, and the total potential takes the form

$$
u(\bar{z})-h \bar{z}=\frac{1}{2 \bar{w}}\left(2 h_{c} \epsilon\right)^{1 / 2} \bar{z}^{2}-\frac{1}{6 \bar{w}^{2}} \bar{z}^{3} .
$$

Consequently, $z_{2}=3 \bar{w}\left(2 h_{c} \epsilon\right)^{1 / 2}$.

With the help of Eq. (22) the extremal action can be presented as

$$
S_{E}=-\frac{\sigma_{0} A_{w}}{\omega} \int_{z_{1}}^{z_{2}} d \bar{z} \sqrt{2[u(\bar{z})-h \bar{z}]} .
$$

Substituting here the potential of Eq. (25) we obtain

$$
S_{E}=-\frac{48}{5} \frac{\sigma_{0} A_{w}}{\omega} \bar{w}^{3 / 2}\left(h_{c} \epsilon\right)^{5 / 4} .
$$

The tunneling rate can be presented as

$$
P=A \exp \left(-B_{0}\right) \text {, }
$$

where the WKB exponent is given by $B_{0}=-S_{E} / \hbar$. In terms of the total number of tunneling spins, $N=M_{0} A_{w} \delta / \mu_{B}$, with the help of Eqs. (14) and (27), we obtain

$$
B_{0}=\frac{48}{5} \frac{\gamma H_{c}}{\omega} \bar{w}^{3 / 2} h_{c}^{1 / 4} \epsilon^{5 / 4} N .
$$

Let us now verify the validity of our assumption that $\left(d \bar{z} / d x_{0}\right)^{2}<<1$, under which Eq. (20) was obtained from Eq. (15). The first integral of Eq. (22) is

$$
\frac{1}{2}\left(\frac{d \bar{z}}{d x_{0}}\right)^{2}=u(\bar{z})-h \bar{z} \text {. }
$$

According to Fig. 2(a) and Eq. (25) it has a maximum at $\bar{z}=z_{0}$,

$$
\left(\frac{d \bar{z}}{d x_{0}}\right)_{\max }^{2}=\frac{8\left(h_{c} \epsilon\right)^{3 / 2}}{3 \sqrt{2} \bar{w}},
$$

which is small in the limit of small $\epsilon$ and $h_{c}$.

Another interesting observation is the universal form of the instanton in the limit of the small barrier. Substituting Eq. (25) into Eq. (30) one obtains

$$
\bar{z}(\tau)=z_{2} / \cosh ^{2}\left(\omega_{0} \tau\right)
$$

where

$$
\omega_{0}=\frac{2\left(2 h_{c} \epsilon\right)^{1 / 4}}{\bar{w}^{1 / 2}} \omega .
$$

Equation (33) describes the imaginary-time motion from 
$\bar{z}=0$ at $\tau=-\infty$ to $\bar{z}=z_{2}$ at $\tau=0$, and then back to $\bar{z}=0$ at $\tau=+\infty$. It corresponds to the classical motion in the inverted potential, $h \bar{z}-u(\bar{z})$.

\section{B. Tunneling in $2+1$ dimensions}

If the area of the domain wall coupled to a planar defect is large, the tunneling will occur via a nucleation process shown in Fig. 1. Once the critical portion of the wall is released due to tunneling, the nucleus will expand and eventually depin the entire wall. Let $X$ be the direction of the anisotropy axis and $l$ be the length of the defect in that direction. The Euclidean action for that problem is

$$
S_{E}=-\frac{\sigma_{0} l \delta}{\omega} \int d^{2} x\left[\frac{1}{2}(\nabla \bar{z})^{2}+u(\bar{z})-h \bar{z}\right],
$$

where $d^{2} x=d x_{0} d x_{2}, \bar{z}=\bar{z}\left(x_{0}, x_{2}\right)$. In this case it is impossible to solve the problem analytically, even for a small barrier. Instead, one can perform a dimensional analysis of Eq. (34), in order to extract the dependence of the WKB exponent on the parameters of the ferromagnetic material. Consider

$$
z^{\prime}=\frac{\bar{z}}{r}, \quad x^{\prime}=\frac{\left(2 h_{c} \epsilon\right)^{1 / 4}}{\bar{w}^{1 / 2}} x
$$

Then, with the help of Eq. (25), at a small barrier, we obtain

$S_{E}=-\frac{\sigma_{0} l \delta}{\omega} \bar{w}^{2} h_{c} \epsilon \int d^{2} x^{\prime}\left[\frac{1}{2}\left(\nabla^{\prime} z^{\prime}\right)^{2}+\frac{1}{2} z^{\prime 2}-\frac{1}{6} z^{\prime 3}\right]$.

The instanton satisfies

$$
\nabla^{\prime 2} z^{\prime}=z^{\prime}-\frac{1}{2} z^{\prime 2}
$$

According Eq. (35), the size of the nucleus is

$$
Y_{n} \sim \frac{\bar{w}^{1 / 2}}{\left(2 h_{c} \epsilon\right)^{1 / 4}} \delta \text {. }
$$

Tunneling via the formation of the nucleus will occur if the size of the defect, $d$, in the $Y$ direction exceeds $Y_{n}$, that is, at

$$
d / \delta>\bar{w}^{1 / 2} /\left(2 h_{c} \epsilon\right)^{1 / 4} .
$$

After the nucleus is formed due to the tunneling, it expands in real time according the classical equation of motion, Eq. (6).

The integral in Eq. (36), evaluated along the instanton trajectory, is a number of the order of unity. Consequently, the factor before the integral gives the estimate of the extremal action. In terms of the number of tunneling spins, $N=M_{0} l Y_{n} \delta / \mu_{B}$, we obtain

$$
B_{0}=2^{1 / 4} k \frac{\gamma H_{c}}{\omega} \bar{w}^{3 / 2} h_{c}^{1 / 4} \epsilon^{5 / 4} N,
$$

where $k \sim 1$ is the value of the integral. Comparison of Eq. (40) with Eq. (29) shows that in terms of $N$ the geometry of the tunneling has little effect on the dependence of $B$ on the parameters of the system. It appears to be quite universal in the limit of low barrier.

\section{THE CROSSOVER TEMPERATURE}

In the absence of dissipation the temperature of the crossover from thermal to quantum regime is given by $k_{B} T_{c}=U_{0} / B_{0}$. It requires, therefore, a calculation of the height of the energy barrier, $U_{0}$. As has been shown above, the limit of the small barrier automatically provides the condition of small derivatives, $|\nabla z|<1$. In this limit the energy of the static configuration of the wall is given by the equation similar to Eq. (20),

$$
E=\sigma_{0} \delta^{2} \int d^{2} x\left[\frac{1}{2}(\nabla \bar{z})^{2}+u(\bar{z})-h \bar{z}\right],
$$

where $d^{2} x=d x_{1} d x_{2}, \nabla=\left(\partial_{1}, \partial_{2}\right)$. For a flat wall studied in Sec. III A, $\nabla \bar{z}=0$ and the height of the barrier is

$$
U_{0}=\sigma_{0} A_{w} u_{0},
$$

where $u_{0}$ is given by Eq. (25) at $\bar{z}=z_{0}$, $u_{0}=\left(\frac{2}{3}\right) \bar{w}\left(2 h_{c} \epsilon\right)^{3 / 2}$. In terms of the number of tunneling spins one obtains

$$
U_{0}=\frac{8 \sqrt{2}}{3} \mu_{B} H_{c} \bar{w} h_{c} \epsilon^{3 / 2} N
$$

Together with Eq. (29) this gives

$$
k_{B} T_{c}=\frac{5 \sqrt{2}}{36} \hbar \omega \bar{w}^{-1 / 2} h_{c}^{1 / 4} \epsilon^{1 / 4} .
$$

Note, that $k_{B} T_{c} \sim \hbar \omega_{0}$, where $\omega_{0}$ is the instanton frequency of Eq. (33).

Let us now turn to the $2+1$ tunneling studied in Sec. III $B$. The energy of the nucleus is

$$
E=\sigma_{0} l \delta \int d x_{2}\left[\frac{1}{2}\left[\frac{d \bar{z}}{d x_{2}}\right]^{2}+u(\bar{z})-h \bar{z}\right] \text {. }
$$

A dimensional analysis similar to that performed in Sec. III B shows that for a small barrier Eq. (45) can be presented as

$E=\sigma_{0} l \delta \bar{w}^{3 / 2}\left(2 h_{c} \epsilon\right)^{5 / 4} \int d y^{\prime}\left[\frac{1}{2}\left[\frac{d z^{\prime}}{d y^{\prime}}\right]^{2}+\frac{1}{2} z^{\prime 2}-\frac{1}{6} z^{\prime 3}\right]$,

where the integral is of the order of unity if taken along the extremal trajectory. The latter satisfies

$$
\frac{d^{2} z^{\prime}}{d y^{\prime 2}}=z^{\prime}-\frac{1}{2} z^{\prime 2}
$$

The solution of this equation,

$$
\bar{z}=\frac{3 \bar{w}\left(2 h_{c} \epsilon\right)^{1 / 2}}{\cosh ^{2}\left(2 y / Y_{n}\right)},
$$

gives the shape of the critical nucleus shown in Fig. 1. Further integration in Eq. (46) gives

$$
U_{0}=\frac{6}{5} \sigma_{0} l \delta \bar{w}^{3 / 2}\left(2 h_{c} \epsilon\right)^{5 / 4}
$$

or the equivalent expression

$$
U_{0}=\frac{24 \sqrt{2}}{5} \mu_{B} H_{c} \bar{w} h_{c}^{1 / 2} \epsilon^{3 / 2} N,
$$


where $N=M_{0} l Y_{n} \delta / \mu_{B}$ is the total number of spins inside the nucleus. Comparing Eq. (50) with Eq. (43) one finds that in terms of $N$ the dependence of the barrier on the parameters is the same for different geometries of tunneling.

Equations (40) and (50) allow one to obtain the crossover temperature for the nucleation process,

$$
k_{B} T_{c}=\frac{12 \times 2^{1 / 4}}{5 k} \hbar \omega \bar{w}^{-1 / 2} h_{c}^{1 / 4} \epsilon^{1 / 4} .
$$

Comparison with Eq. (44) again demonstrates a universal dependence of $T_{c}$ on the parameters. The only modeldependent parameter here is the width of the barrier, which one can associate with the size of the defect. Other parameters can be found from independent experiments.

\section{THE PREEXPONENTIAL FACTOR}

Let us now estimate the prefactor $A$ in the expression for the tunneling rate, $P=A \exp \left(-B_{0}\right)$. It is determined by the contribution to the path integral of tunneling trajectories which are small perturbations of the instanton. The general expression for $A$ in the case of the flat wall tunneling through a planar defect is ${ }^{15,16}$

$$
A=\left(\frac{B_{0}}{2 \pi}\right)^{1 / 2}\left(\frac{\operatorname{det} \hat{K}_{0}}{\operatorname{det}^{\prime} \hat{K}}\right)^{1 / 2} \text {. }
$$

Here det' means exclusion of the zero mode corresponding to the translational time invariance of the instanton,

$$
\hat{K}_{0}=-\partial_{\tau}^{2}+\omega_{1}^{2}, \quad \hat{K}=-\partial_{\tau}^{2}+\omega^{2} u^{\prime \prime}\left(\bar{z}_{i}\right),
$$

where $\omega_{1}=\omega_{0} / 2$ is the frequency of small oscillations near the minimum of the potential at $\bar{z}=z_{1}$ [Fig. 2(a)], $\omega_{0}$ is the instanton frequency given by Eq. (33), and $\bar{z}_{i}=z_{2} / \cosh ^{2}\left(\omega_{0} \tau\right)$ is the instanton, Eq. (32).

The computation of $A$ is, in general, a difficult problem. It can be done rigorously, however, in the case of a small barrier, which is of primary interest here. With the help of Eqs. (25) and (32) we obtain

$$
u^{\prime \prime}\left(z_{i}\right)=\frac{1}{4}\left(1-\frac{3}{\cosh ^{2}\left(\omega_{0} \tau\right)}\right) \text {. }
$$

Each determinant in Eq. (52) is the product of eigenvalues of operators in Eq. (53). The problem, therefore, reduces, to the solution of

$$
\begin{aligned}
& \left(-\partial_{\tau}^{2}+\omega_{1}^{2}\right) \phi_{n}=\lambda_{n} \phi_{n} \\
& {\left[-\partial_{\tau}^{2}+\omega_{1}^{2}\left(1-\frac{3}{\cosh ^{2}\left(\omega_{0} \tau\right)}\right)\right] \psi_{n}=\mu_{n} \psi_{n} .}
\end{aligned}
$$

Exclusion of the zero mode, $\mu_{n}=0$, leads to the propor- tionality of the ratio of determinants in Eq. (52) to $\omega_{0}^{2}$. Evaluating this ratio by standard methods ${ }^{4}$ one obtains

$$
\begin{aligned}
\frac{\operatorname{det}\left(-\partial_{\tau}^{2}+\omega_{1}^{2}\right)}{\operatorname{det}^{\prime}\left[-\partial_{\tau}^{2}+\omega_{1}^{2}\left(1-3 / \cosh ^{2}\left(\omega_{0} \tau\right)\right]\right.} & =\frac{\Pi_{n} \lambda_{n}}{\Pi_{n}^{\prime} \mu_{n}} \\
& =15 \omega_{0}^{2} .
\end{aligned}
$$

This gives for the prefactor

$$
A=\left(\frac{15}{2 \pi}\right)^{1 / 2} B_{0}^{1 / 2} \omega_{0},
$$

where $B_{0}$ and $\omega_{0}$ are given by Eqs. (29) and (33), respectively.

Observation of tunneling requires a not very large $B$. Consequently, the value of the prefactor $A$ for any reasonable experiment will be somewhere between $\omega_{0}$ and $10 \omega_{0}$. Based upon dimensional arguments, one can see that this must be the case for any geometry of the tunneling, including the nucleation process studied above.

\section{DISSIPATION}

Up to this moment we have completely neglected interaction of the domain wall with microscopic degrees of freedom like electrons, phonons, magnons, etc. The effect of these interactions on the tunneling rate must be computed along the lines of the approach developed by Caldeira and Leggett. ${ }^{5}$ The idea is to write the action which includes all such interactions and then, integrating over microscopic variables, to obtain the effective action in terms of the macroscopic variable only. Contributions of various dissipation mechanisms are discussed below.

\section{A. Conduction electrons}

One may directly apply the results of Ref. 5 to the Ohmic coupling of $\mathbf{M}(\mathbf{x}, t)$ with conducting electrons, assuming $\mathbf{M}(\mathbf{x}, t)$ couples linearly to the electronic coordinates. The heating rate in a ferromagnet is given by

$$
\frac{d Q}{d t}=\frac{1}{2} \eta \int d^{3} R \dot{\mathbf{M}}^{2} \sim \frac{\eta M_{0}^{2}}{\delta} \int d x d y \dot{z}^{2},
$$

where $z(x, y, t)$ describes propagation of the domain wall; $\eta$ is a constant. The methods of Caldeira and Leggett ${ }^{5}$ imply that this Ohmic dissipation adds a contribution

$$
S_{\mathrm{dis}}=-\frac{\eta \delta M_{0}^{2}}{4 \pi} \int d x d y \int d x_{0} \int d x_{0}^{\prime} \frac{\left[\bar{z}\left(x_{0}\right)-\bar{z}\left(x_{0}^{\prime}\right)\right]^{2}}{\left(x_{0}-x_{0}^{\prime}\right)^{2}}
$$

to the tunneling action (21). Then the total Euclidean action becomes

$$
S_{\mathrm{tot}}=-\frac{\sigma_{0}}{\omega} \int d x d y \int d x_{0}\left[\frac{1}{2}\left[\frac{d \bar{z}}{d x_{0}}\right]^{2}+u(\bar{z})-h \bar{z}+\beta \int d x_{0}^{\prime} \frac{\left[\bar{z}\left(x_{0}\right)-\bar{z}\left(x_{0}^{\prime}\right)\right]^{2}}{\left(x_{0}-x_{0}^{\prime}\right)^{2}}\right]
$$


where

$$
\beta=\frac{\eta M_{0}^{2} v_{0}}{4 \pi \sigma_{0}}
$$

determines the relative contribution of this dissipation to the WKB exponent. In terms of the parameters, $\beta$ is of the order of the relative width of the ferromagnetic resonance. This suggests that materials with narrow FMR are better candidates for the observation of quantum tunneling of domain walls.

\section{B. Magnons}

Various branches of spin waves, including bulk magnons and magnons in the wall, may, in principle, contribute to the dissipation. The domain wall is a soliton of the nonlinear equation of motion for the magnetization, Eq. (6). As has been observed by Stamp, ${ }^{4}$ the problem of the macroscopic quantum tunneling of solitons is essentially different from the problem studied by Caldeira and Leggett. Indeed, any soliton, being a solution of a nonlinear differential equation, does not have, by definition, a linear coupling with the excitations of the field describing the soliton. Consequently, the interaction of the domain wall with magnons starts with a term of the second order in magnon variables. Moreover, as shown in Ref. 4, for the case of planar walls, the important coupling between the wall and the magnons is not even the second-order one, but the third-order coupling.

The full details of the discussion of the dissipative effects of magnons are, therefore, rather involved; ${ }^{17}$ what we wish to show here is how the calculations for a planar wall can be adapted in a completely straightforward way for a curved wall, provided only that the wall curvature is small (as indeed we have been assuming until now). To do this we shall show how the main steps of the calculation for a planar wall have their counterpart for a curved wall. To simplify the problem, we will keep the uniaxial anisotropy $K_{\|}$and the dipole term $2 \pi M_{0}^{2}$ in the formulas of this section, but not the transversal anisotropy $K_{\perp}$. The presence of $K_{\perp}$ would not change any of our conclusions on the effect of dissipation.

In order to determine the effects of various magnon couplings, we must first perform the maneuver, familiar from soliton theory, of quantizing the magnetic fluctuations in the presence of the wall. Thus for a simple Bloch wall, the magnons no longer satisfy a simple wave equation, but are the eigenmodes of a more complicated Hamiltonian

$$
\mathscr{H}=\mathscr{H}_{m}^{(2)}+\mathscr{H}_{m}^{(3)}+\cdots
$$

in which the superscripts label two-magnon, threemagnon, etc., terms; for a stationary wall one has

$$
\mathscr{H}_{m}^{(2)}=-\int d^{3} r\left\{\Delta_{0}^{2} \delta^{2}|\nabla b(\mathbf{r}, t)|^{2}+\left[\Delta_{0}\left[1-2 \operatorname{sech}^{2}\left[\frac{z-Z}{\delta}\right)\right]+4 \pi \hbar \gamma M_{0}\right] b^{+}(\mathbf{r}, t) b(\mathbf{r}, t)\right\}
$$

for the second-order term, and

$$
\mathscr{H}_{m}^{(3)}=i \int d^{3} r \hbar^{2} \gamma^{2} K_{\|}\left(\frac{32 s}{a_{0}^{3}}\right)^{1 / 2} \operatorname{sech}\left(\frac{z-Z}{\delta}\right)\left[\tanh \left(\frac{z-Z}{\delta}\right)-\delta \nabla_{r}\right]\left[b^{+}(\mathbf{r}, t)-b(\mathbf{r}, t)\right] b^{+}(\mathbf{r}, t) b(r, t)
$$

for the third-order term (see Refs. 4 and 17; there were a number of misprints in Ref. 4). In these equations $\Delta_{0}$ is the energy of the bulk magnon at zero momentum [see. Eq. (66) below], $Z(x, y)$ describes the wall, $b(\mathbf{r}, t)$ and $b^{\dagger}(\mathbf{r}, t)$ are the magnon fields; $(r, t)=(x, y, z, t)$. These magnon fields are produced by the usual Holstein-Primakoff transformation, but again they refer now to the magnons quantized about the soliton solution. Thus, if we invent a triad of vectors $\left(\mathbf{e}_{1}, \mathbf{e}_{2}, \mathbf{e}_{3}\right)$ in which $\mathbf{e}_{3}$ is directed along the local magnetization axis $\mathbf{M}(\mathbf{r}, t)$, then we have fluctuations about this magnetization (the "fast modes") described by the fluctuation field $\delta \mathbf{M}(\mathbf{r}, t)$, where

$$
\begin{aligned}
& \delta M_{+}(\mathbf{r}, t)=\delta M_{1}+i \delta M_{2}=\left(4 \hbar \gamma M_{0}\right)^{1 / 2}\left[1-\frac{2 \hbar \gamma}{M_{0}} b^{\dagger}(\mathbf{r}, t) b(\mathbf{r}, t)\right]^{1 / 2} b(\mathbf{r}, t), \\
& \delta M_{-}(\mathbf{r}, t)=\delta M_{1}-i \delta M_{2}=\left(4 \hbar \gamma M_{0}\right)^{1 / 2} b^{\dagger}(\mathbf{r}, t)\left[1-\frac{2 \hbar \gamma}{M_{0}} b^{\dagger}(\mathbf{r}, t) b(\mathbf{r}, t)\right]^{1 / 2}, \\
& \delta M_{3}(\mathbf{r}, t)=-4 \hbar \gamma b^{\dagger}(\mathbf{r}, t) b(\mathbf{r}, t) .
\end{aligned}
$$

Thus the field $b(\mathbf{r}, t)$ is not the usual magnon field, because the triad $\left(e_{1}, e_{2}, e_{3}\right)$ rotates as we move around. It is important to notice that Eqs. (62)-(65) imply that we may not use the Landau-Lifshits-Gilbert (LLG) equation to analyze wall-magnon dissipation processes. This is because $|\boldsymbol{M}(\mathbf{r}, t)|$ is not conserved by many of the magnon processes involved. This fact is well known in the theory of ferromagnetic resonance, although it does not seem to have prevented a large number of papers on dissipation from using the LLG equation to treat ferromagnetic insulators. This point becomes all the more important when one sees that Ohmic dissipation in magnetic insulators 
comes entirely from processes in which $|\mathbf{M}|$ is not conserved (see below).

The spectrum of magnetic fluctuations in the presence of the wall can be obtained from $\mathscr{H}_{m}^{(2)}$, and for the planar wall one finds a set of "bulk modes," with spectrum

$$
\omega_{k}^{2}=\frac{4 \hbar^{2} \gamma^{2}}{M_{0}^{2}}\left(A_{e} k^{2}+K_{\|}\right)\left(A_{e} k^{2}+K_{\|}+2 \pi M_{0}^{2}\right),
$$

where $\mathbf{k}$ is the three-dimensional wave vector, and "wall modes," with spectrum

$$
\epsilon_{q}^{2}=\frac{4 \hbar^{2} \gamma^{2}}{M_{0}^{2}} A_{e} q^{2}\left(A_{e} q^{2}+2 \pi M_{0}^{2}\right)
$$

where $q$ is a two-dimensional wave vector, confined to the wall plane. The wall modes are the second-quantized version of the semiclassical small oscillations of the wall, and are, thus, confined to the wall region; writing $b(\mathbf{r}, t)$ as the sum of a wall contribution $b_{w}(\mathbf{r}, t)$ and a bulk contribution $b_{b}(\mathbf{r}, t)$, one has

$b(\mathbf{r}, t)=\sum_{\mathbf{q}} \Phi_{\mathbf{q}}(\mathbf{r}, t) b_{w}(\mathbf{r}, t)+\sum_{\mathbf{k}} \Psi_{\mathbf{k}}(\mathbf{r}, t) b_{b}(\mathbf{r}, t)$,

where

$$
\begin{aligned}
& \Phi_{\mathbf{q}}(\mathbf{r}, t)=\frac{e^{\imath \mathbf{q} \cdot \rho}}{\left(A_{w} \delta\right)^{1 / 2}} \operatorname{sech}\left[\frac{z-Z(x, y, t)}{\delta}\right], \\
& \Psi_{\mathbf{k}}(\mathbf{r}, t)=\frac{e^{i \mathbf{k} \cdot \mathbf{r}}}{\Omega} \frac{\tanh \left[\frac{z-Z(x, y, t)}{\delta}\right)-i k_{z} \lambda}{\left(1+k_{z}^{2} \delta^{2}\right)^{1 / 2}} .
\end{aligned}
$$

Here $\rho$ is a two-dimensional radius vector in the wall plane, and we have allowed the wall coordinate $Z(x, y, t)$ to be time dependent. Notice that bulk magnons are excluded from the wall except at high energies, such that $k_{z} \delta>1$.

Up to this point, we have simply altered the formulas for wall-magnon coupling to take account of the shape of the wall. This is in the spirit of the approximation described in Sec. II, where we dropped terms $\sim\left(\delta / R_{0}\right)^{2}$ in the Slonczewski equations for a gently curved wall. To calculate the dissipative effects of this coupling we need to calculate the transfer of energy and momentum be-
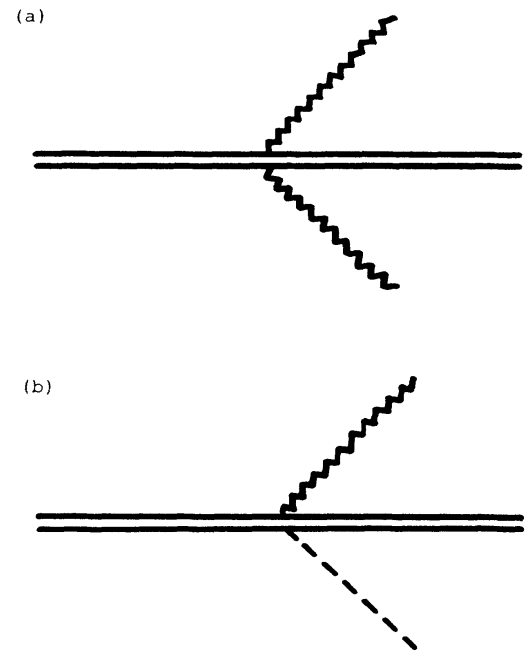

FIG. 3. Two magnon processes which give a non-Ohmic contribution to wall dissipation. The wall is represented by the thick double line; bulk magnons are represented by a jagged line, and wall magnons are represented by a hatched straight line. The term in (a) is the leading term; the term in (b) is $\sim O\left(\delta / R_{0}\right)$ and we have ignored it.

tween the wall and the magnons when the wall is moving, and this involves two related approximations: (i) we are going to ignore momentum transfer perpendicular to the $z$ axis and (ii) we assume that the magnon bath is in equilibrium (at temperature $T$ ) and that this equilibrium is undisturbed by the wall motion. The first approximation ignores corrections $\sim\left(\delta / R_{0}\right)^{2}$. The second approximation is more difficult to justify formally, but seems physically reasonable if $|\dot{Z}|<<v_{0}$. It is well known that as $|\dot{Z}|$ approaches $v_{0}$, this assumption will break down but we are working in the low-velocity limit.

Turning now to the calculation of energy-momentum transfer, we proceed as follows for the curved wall. Just as for the planar wall, it is convenient to set up the calculation using time-dependent perturbation theory in which the moving wall perturbs the magnon bath and causes transitions between bath states $|j\rangle$ and $|i\rangle$, governed by the transition amplitude

$$
T_{i j}(t)=\int d^{3} r \exp \left[\frac{i}{\hbar} \int_{-\infty}^{t} d t^{\prime} E_{i}\left(t^{\prime}\right) \frac{\langle i|\partial \hat{V} / \partial t| j\rangle}{E_{i}(t)-E_{j}(t)}\right] \exp \left[-\frac{i}{\hbar} \int_{-\infty}^{t} d t^{\prime} E_{j}\left(t^{\prime}\right)\right],
$$

where $\hat{V}$ is the perturbation operator of the wall on the magnon bath, and $E_{i}(t)$ is the instantaneous adiabatic energy of the bath mode $|i\rangle$. Now consider a wall with a velocity profile $\dot{Z}(x, y, t)$; Eq. (71) then becomes

$$
T_{i j}(t)=\int d x d y \dot{Z}(x, y, t) \frac{e^{i\left(E_{i}-E_{j}\right) t}}{E_{i}-E_{j}}\langle i|\partial \hat{V} / \partial z| j\rangle \text {. }
$$

We now wish to show that, in the same way as for a planar wall, the third-order coupling between the wall surface and the magnons gives the leading contribution to the dissipation. First we deal with two-magnon processes (Fig. 3). These give a friction coefficient

$$
\delta \Gamma_{2}\{\dot{Z}\}=\frac{2 \pi}{\hbar} \int d x d y \sum_{k, k^{\prime}} \frac{\left|\mathcal{M}_{k k^{\prime}}^{(2)}\right|^{2}}{\omega_{k}-\omega_{k^{\prime}}}\left(n_{k}-n_{k^{\prime}}\right) \delta\left(\omega_{k}-\omega_{k^{\prime}}+\left(\mathbf{k}-\mathbf{k}^{\prime}\right) \cdot \dot{\mathbf{Z}}(x, y)\right),
$$


where $\mathcal{M}_{k k^{\prime}}^{(2)}$ is the two-magnon matrix element derived from $\left\langle\mathbf{k}|\partial \widehat{V} / \partial z| \mathbf{k}^{\prime}\right\rangle$. The energies $\omega_{k}, \omega_{k^{\prime}}$ are bulk magnon energies, and $n_{k}=n\left(\omega_{k}\right)$ is the Bose distribution function.

Calculation of (73) yields a frictional coefficient which is highly nonlinear in velocity,

$$
\delta \eta_{2}\{\dot{Z}, T\} \sim \int d x d y \dot{Z}^{4}(x, y) e^{-\Delta_{0} / k_{B} T} .
$$

At low velocities this friction is negligible. However at third-order, the important Ohmic processes come in. The critical term is that described by the graph in Fig. 4, which gives a contribution to the friction coefficient of

$$
\delta \Gamma_{3}\{\dot{Z}\}=\frac{2 \pi}{\hbar} \int d x d y \sum_{k k^{\prime} q} \frac{\left|\mathcal{M}_{k k^{\prime} q}^{(3)}\right|^{2}}{\omega_{k}-\omega_{k^{\prime}}-\epsilon_{q}} F\left\{n_{j}\right\} \delta\left(\omega_{k}-\omega_{k^{\prime}}-\left(\mathbf{k}-\mathbf{k}^{\prime}-\mathbf{q}\right) \cdot \dot{\mathbf{Z}}(x, y)\right),
$$

where the statistical factor $F\left\{n_{j}\right\}$ is

$$
F\left\{n_{j}\right\}=\left[n_{k}\left(1+n_{k^{\prime}}\right)\left(1-n_{q}\right)-n_{k^{\prime}} n_{q}\left(1+n_{k}\right)\right]
$$

and $\boldsymbol{M}_{k k^{\prime} q}^{(3)}$ describes the conversion of a bulk magnon into another bulk magnon, plus a wall magnon, with the transfer of momentum $\left(\mathbf{k}-\mathbf{k}^{\prime}-\mathbf{q}\right)$ to the wall. Calculation of (75) gives the following result for temperatures $k_{B} T \leq \Delta_{0}$ :

$\delta \eta_{3}(\dot{Z}, T)=\int d x d y \frac{1}{16 \pi^{2} \gamma^{2} \delta^{3}}\left(\frac{k_{B} T}{\Delta_{0}^{2}}\right) e^{-\Delta_{0} / k_{B} T}$

We see here that $\delta \eta_{3}$ is independent of $\dot{Z}$, and hence the dissipation process is Ohmic. It can be shown ${ }^{4,17}$ that fourth-order and higher processes give terms of higher order in $\left(k_{B} T / \Delta_{0}\right)$ than (77), and so we shall ignore them. Thus $\delta \eta_{3}(T)$ is the most important dissipation term coming from magnons; note that it involves a process in which $|\mathbf{M}(\mathbf{r}, t)|$ is not conserved, and so the dissipation term cannot be described by the LLG equation (6).

Now the problem is how to find the effects of this dissipation on tunneling. We cannot directly apply the Caldeira-Leggett scheme to our problem, because the crucial coupling is third-order in the environmental variables; the Caldeira-Leggett framework is based on a set of environmental couplings which are linear in the environmental coordinates. However for Ohmic dissipation it turns out we can circumvent this problem. We define a function

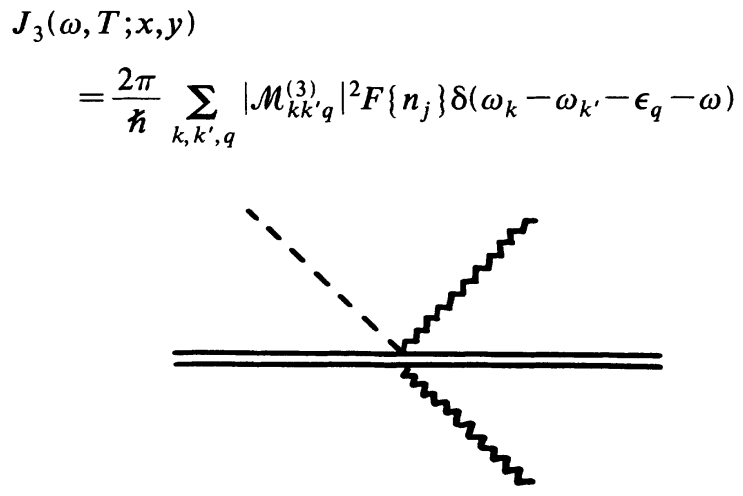

FIG. 4. The leading third-order process, which gives rise to Ohmic dissipation of wall motion; the wall interacts with two bulk magnons and one wall magnon (see text). Other processes are of higher order in $\left(T / \Delta_{0}\right)$. and now observe that provided we may ignore backscattering processes in which magnons are reabsorbed by the wall, the equation of motion of the domain wall will be governed by the Slonczewski equations with an extra dissipative term acting on $Z(\omega, x, y)$ of the form $K_{3}(\omega, T ; x, y) Z(x, y)$, where

$$
K_{3}(\omega, T ; x, y)=\frac{2 \omega^{2}}{\pi} \int_{0}^{\infty} d \epsilon g(\epsilon) \frac{J_{3}(\epsilon, T ; x, y)}{\epsilon\left(\epsilon^{2}-\omega^{2}\right)},
$$

where $g(\epsilon)$ is a "cutoff" function, which smoothly cuts off the linear behavior of $J_{3}(\omega)$ at large $\omega$; the form of $J_{3}(\omega, T ; x, y)$ is just

$$
\begin{aligned}
J_{3}(\omega, T ; x, y) & =\frac{\omega}{16 \pi^{2} \gamma^{2} \delta^{3}}\left[\frac{k_{B} T}{\Delta_{0}^{2}}\right] e^{-\Delta_{0} / k_{B} T} \\
& \equiv \omega \eta_{3}(x, y ; T) .
\end{aligned}
$$

Thus we have arrived at a dynamic equation for the wall, in which the Slonczewski equations have an Ohmic dissipation term added to them; for if we make a time-Fourier transform of Eq. (80), it just gives a term $\eta_{3} \dot{Z}(x, y ; t)$ in the Slonczewski equations, with $\eta_{3}$ coming from threemagnon processes.

Now if $|\dot{Z}(x, y)| \ll<v_{0}$ and $\omega<v_{0} / \delta$, we may carry all of usual ideas of Leggett ${ }^{18}$ over to our case, provided we now regard the bath as being a set of temperaturedependent modes (each of which describes a triplet of magnons) which are coupled to the wall via a temperature-dependent coupling function; all of this is encapsulated in Eq. (80). Furthermore, if we are interested in tunneling over a length scale $\sim \epsilon^{1 / 2} \delta$ which is much less than the other length scales in the problem (as in the case here), this coupling can be treated as being linear in $Z(x, y)$. Then, quite remarkably, the entire effect of the wall-magnon interactions can be absorbed into the nonlocal contribution to the imaginary-time action of the form (60), with the following parameter $\beta$ :

$$
\beta=\frac{\hbar \omega k_{B} T}{4 \pi \Delta_{0}^{2}} e^{-\Delta_{0} / k_{B} T}
$$

This contribution is of exactly the Caldeira-Leggett form for Ohmic coupling; we notice that while it is nonlocal in imaginary time, it is local in the spatial coordinates of the wall. This latter result is only true for small wall curvature.

Now we see that for $k_{B} T \ll \Delta_{0}$, we may always neglect 
dissipation from wall-magnon coupling. However, at $T \sim T_{c}$ this is not necessarily true. Generally, $\hbar \omega$ and $\Delta_{0}$ have the same order of magnitude [see Eqs. (19) and (66)], unless very anisotropic crystals are considered. The crossover temperature (see Sec. IV) is $\sim\left(h_{c} \epsilon\right)^{1 / 4} \hbar \omega$. Consequently, at $T \sim T_{c}$ the relative contribution of the magnon dissipation into the WKB exponent is $\sim\left(h_{c} \epsilon\right)^{1 / 4} \exp \left[-1 /\left(h_{c} \epsilon\right)^{1 / 4}\right]$. Because of the conditions $h_{c}<1, \epsilon<1$, this contribution cannot be large but may, in principle be of the order of one. It is small, however, in the case of a small barrier, $h_{c} \epsilon<<1$, provided that the most significant dissipation mechanism in insulators does not affect the possibility to observe quantum tunneling of domain walls.

\section{Phonons}

The problem of phonon dissipation turns out to be rather subtle. At first glance one might think that since the magnetoelastic coupling in most magnets is rather small, the effect of phonons should be rather negligible. This guess is certainly confirmed at the level of linear coupling between phonons and the domain wall, as it was for magnetic grain tunneling in Ref. 13. The argumentation is somewhat similar. In both, the coupling chosen is embodied in the action

$S_{\mathrm{ph}}=\int d t \int d^{3} x\left\{\frac{1}{2} \rho \dot{\mathbf{u}}^{2}-\frac{1}{2} \lambda_{i k l m} u_{i j} u_{l m}-a_{i k l m} u_{i j} M_{l} M_{m}\right\}$,

where $\mathbf{u}$ is the phonon displacement field, $u_{i k}=\frac{1}{2}\left(\partial_{i} u_{k}+\partial_{k} u_{i}\right)$ is the strain tensor, $\hat{\lambda}$ and $\hat{a}$ are the elastic and magnetoelastic tensors, respectively, and $\rho$ is the mass density of the material. As Garg and Kim showed, for magnetic grains this leads to a CaldeiraLeggett spectral function $J(\omega) \propto \omega^{3}$, which is infrared weak; moreover, it is small anyway, as one might expect from the fact that the adiabatic effects of this coupling are also weak. They lead to a relative change of the order of $10^{-5}$ in the effective moment of inertia associated with the subbarrier rotation of $\mathbf{M}$ and to the same change in the wall mass. Indeed, if the tunneling frequency $\omega_{0}$ is small in comparison with $s / \delta$, where $s$ is the speed of sound, then the elastic deformation of the solid due to magnetiostriction simply follows the rotating magnetization as the wall moves in imaginary time. For $\omega_{0} \sim 10^{10}$ $\mathrm{s}^{-1}, s \sim 10^{5} \mathrm{~cm} / \mathrm{s}$, and $\delta \sim 10^{-6} \mathrm{~cm}$ it becomes a rather good approximation. The imaginary-time dependence of the elastic deformation that adiabatically follows the motion of the wall is $u(\tau)=u[Z-Z(\tau)]$, where $Z(\tau)$ is the instanton solution of Sec. III. Substituting this into Eq. (82), we obtain that the magnetoelastic interaction contributes the term $m^{\prime} \sim \rho u_{0}^{2} / \delta$ to the effective mass of the wall in Eq. (13). Here $u_{0}$ is the deformation of the crystal, due to the magnetostriction, inside the wall. It can be estimated as $u_{0} \sim \Delta \delta$, where $\Delta \sim a M_{0}^{2} / 2 E$ is a small dimensionless constant, $E$ heing Young's modulus. Consequently, the relative increase in the wall mass due to the interaction with phonons is $m^{\prime} / m \sim \Delta^{2} \rho v_{0}^{2} \delta / \sigma_{0}$. The constant $\Delta$ is typically $10^{-4}$, which gives $m^{\prime} / m \sim 10^{-5}$.
If one looks at the phonon effects on wall dissipation, the argument is similar, except that one now finds, in a similar way to the work of Wada and Schrieffer, ${ }^{19}$ that the main contribution to the dissipation is apparently from two-phonon elastic processes. Again, the details of this process ${ }^{4,17}$ are not strongly affected by wall curvature, and the dissipative effects are very small $\left(\propto T^{3}\right)$. Nevertheless, we are not entirely sure that this is the end of the story. It has recently been shown by one ${ }^{20}$ of us that in the grain-tunneling problem, inelastic two-phonon processes will dominate over the one-phonon process considered by Garg and $\mathrm{Kim}$, since they give an Ohmic dissipation $[J(\omega) \propto \omega]$. Thus the possibility of Ohmic dissipation arising from inelastic two-phonon interactions with the domain wall remains to be considered.

\section{Photons}

To complete our picture we consider also the electromagnetic radiation produced by the domain-wall tunneling in magnetic insulators. Since the tunneling is described quasiclassically, the analysis of the radiation problem can be also based upon a classical formula,

$$
i=\frac{2}{3 c^{3}} \ddot{\mathbf{M}}^{2}
$$

for the intensity of electromagnetic waves radiated by the rotating magnetic dipole $\mathbf{M}$. For the process of tunneling via nucleation studied in Sec. III B, the rotating magnetic moment is the moment of the nucleus. Substituting the corresponding instanton solution into Eq. (83) and integrating over the imaginary time from $-\infty$ to $+\infty$, one obtains for the total radiated energy

$$
e \sim \frac{M_{0}^{2} V^{2} \omega_{0}^{3}}{c^{3}},
$$

where $V$ is the volume of the nucleus. This energy is much less than $\hbar \omega_{0}$, suggesting that the coupling to photons is irrelevant.

\section{E. Dissipation in terms of mobility}

The above considerations show that the most important dissipation effects (from conducting electrons in metals, and from magnons in insulators) are Ohmic. For that reason it is useful to express the relative contribution of the dissipation in terms of the mobility of the domain wall. The latter can be easily obtained from a macroscopic experiment.

The mobility of the domain wall with respect to the magnetic field, $\mu_{H}$, is defined as

$$
v=\mu_{H} H \text {. }
$$

One can connect it with a more conventional mobility, $\mu_{F}$, with respect to the force, $F$, on the unit area of the wall, $v=\mu_{F} F$. Since $F=2 M_{0} H$ we get $\mu_{H}=2 M_{0} \mu_{F}$. According to Eq. (4), the characteristic frequency associated with the dissipation is $v=1 / \mu_{F} m_{0}$, where $m_{0}$ is the mass of the unit area of the wall, $m_{0}=\sigma_{0} / v_{0}^{2}$. Then Eq. (5) provides a rough estimate of the effect of dissipation on tunneling, 


$$
B=B_{0}\left(1+\frac{M_{0} v_{0}^{2}}{\mu_{H} \sigma_{0} \omega_{0}}\right) .
$$

The quantitative significance of dissipation is discussed in the next section.

\section{DISCUSSION}

An experiment that would study tunneling of a singledomain wall through a planar defect may be somewhat similar to experiments on tunneling in Josephson junctions. In the magnetic case the junction can be made of a layer of the material which is different from the bulk. The material of the junction should be selected such that it pins the wall. Modern evaporation techniques allow one to obtain thicknesses of the junction (defect) as small as one atomic monolayer. The critical field $H_{c}$ will, in general, depend on the thickness $w$ and the material of the junction. The width of the potential well, produced by the junction itself, can hardly be significantly less than the domain wall width. Correspondingly, one should expect that the parameter $\bar{w}$ in our formulas is of the order of one for $w<\delta$, and is roughly given by $w / \delta$ for $w>\delta$. For the narrow junction, from Eqs. (40) and (51), we have

$$
k_{B} T_{c} \sim \hbar \omega\left(h_{c} \epsilon\right)^{1 / 4}
$$

for the crossover temperature, and

$$
A \sim B^{1 / 2} k_{B} T_{c} / \hbar
$$

and

$$
B \sim \frac{\gamma H_{a}}{\omega}\left(h_{c} \epsilon\right)^{5 / 4} N,
$$

respectively, for the prefactor and the WKB exponent of the tunneling rate, $P=A \exp (-B) ; \quad \omega=v_{0} / \delta$, $h_{c}=H_{c} / H_{a}, \epsilon=1-H / H_{c}$. These formulas allow one to estimate the effect based upon the data on the coercive field $H_{c}$, the anisotropy field $H_{a}$, and the parameters of the domain wall: the limiting velocity $v_{0}$ and the thickness $\delta$. Note that the dependence of $T_{c}, A$, and $B$ on $\epsilon$ coincides with that obtained by Stamp. ${ }^{4}$ Equation(87) shows that the dependence of $T_{c}$ on $H_{c}$ and $\epsilon$ is rather weak, and $T_{c}$ is mostly determined by the frequency $\omega$. In the limit of large transverse anisotropy $K_{\perp}$, Eq. (19) gives $\hbar \omega \sim\left(4 \mu_{B} / M_{0}\right)\left(K_{\|} K_{\perp}\right)^{1 / 2}$. Up to a numerical factor, this coincides with the crossover temperature obtained by the exact solution of the equations of micromagnetic theory for tunneling of magnetization in single-domain particles ${ }^{2}$ and quantum nucleation of magnetic bubbles. ${ }^{3}$ For an uniaxial ferromagnet, $K_{\perp}$ in Eq. (19) must be replaced by the magnetic dipole energy $2 \pi M_{0}^{2}$. In the limit of large uniaxial anisotropy, $K_{\|} \gg 2 \pi M_{0}^{2}$, this gives $\hbar \omega=4 \pi \mu_{B} M_{0}$. This energy itself corresponds to the temperature $0.1-1 \mathrm{~K}$ for typical values of $M_{0}, M_{0} \sim 10^{2}-10^{3} \mathrm{emu} / \mathrm{cm}^{3}$. Let us now estimate how many spins can participate in the tunneling process. For $H_{c} \sim 10 \mathrm{Oe}$ and $H_{a} \sim 10^{5} \mathrm{Oe}, B$ of Eq. (88) is of the order of $10^{-3} \epsilon^{5 / 4} N$. This suggests that even at $\epsilon \sim 0.1$, that is without a very fine tuning of the magnetic field, as many as $10^{6}$ spins can coherently tunnel through the energy barrier.

The relative contribution of the dissipation to the tunneling rate is of the order of

$$
\beta_{a} \frac{\gamma \delta}{\mu_{H}}\left(h_{c} \epsilon\right)^{-1 / 4},
$$

where $\beta_{a}$ is a factor determined by the structure of the magnetic anisotropy. (For a rhombic crystal $\beta_{a}=\left[\left(1+K_{1} / K_{\|}\right)^{1 / 2}-1\right]$. ) For typical numbers, $\beta_{a} \sim 10, \delta \sim 10^{-6} \mathrm{~cm}, \mu_{H} \sim 10^{2} \mathrm{~cm} / \mathrm{s}$ Oe, $h_{c} \sim 10^{-4}$, $\epsilon \sim 0.1$, this contribution is of the order of one. We may, therefore, conclude that in materials with low mobility of domain walls dissipation may significantly affect tunneling. In the case of high mobility, our formulas for undamped tunneling should provide a good estimate of the tunneling rate.

To date all experiments on the low-temperature behavior of domain walls have been performed in bulk samples. In this case the rate of quantum magnetic relaxation is determined by the statistical average over a large number of individual tunneling events for many domain walls. Although this problem is much more complicated than studied here, we believe that formulas (87) and (88) provide a rough estimate of the effect. For a statistical contribution of barriers, one can hardly expect any smallness in Eqs. (87) and (88) associated with the parameter $\epsilon$. In materials with strong uniaxial anisotropy $k_{B} T_{c}$ $\sim 4 \pi \mu_{B} M_{0} h_{c}^{1 / 4}$ would be, therefore, a reasonable estimate of $T_{c}$.

A few experiments ${ }^{11,12}$ have been recently performed to address specifically the question of a possible quantum tunneling of magnetization at low temperature. A temperature-independent relaxation rate has been observed in $\mathrm{Tb}_{0.5} \mathrm{Ce}_{0.5} \mathrm{Fe}_{2}\left(M_{0} \sim 200 \mathrm{emu} / \mathrm{cm}^{3}, H_{a} \sim 5 \times 10^{5}\right.$ Oe, $H_{c} \sim 5 \times 10^{3} \mathrm{Oe}$ ) below $T \sim 1 \mathrm{~K}$ (Ref. 11 ), in qualitative agreement with our theoretical estimate. Note that some experiments ${ }^{12}$ may also be explained by quantum nucleation of magnetic bubbles ${ }^{3}$ or by the uniform subbarrier rotation of the magnetization in small grains. ${ }^{2} \mathrm{~A}$ detailed comparison between the theory and experiment on quantum tunneling of magnetization remains a challenging task. In this connection the experiment with a single-wall tunneling through a flat junction would be of great interest. One should also think about the possibility to test the effect of dissipation on tunneling in such an experiment. According to Eqs. (86) and (89) it may become noticeable in materials with very low mobility of domain walls. Of course, the extraction of the contribution of the dissipation from experimental data would require changing the mobility (e.g., by changing the concentration of impurities) without a significant change in other parameters of the tunneling.

\section{ACKNOWLEDGMENTS}

At different stages of this work, the authors benefited from discussions with Bernard Barbara, Leon Gunther, Myriam Sarachik, Javier Tejada, and Alex Vilenkin. E.C. acknowledges support from NSF Grant No. DMR9024250. 
${ }^{1}$ E. M. Chudnovsky, Zh. Eksp. Teor. Fiz. 77, 2157 (1979) [Sov. Phys. JETP 50, 1035 (1979)]; this work formulated a general approach to the tunneling of magnetization but failed to compute the correct tunneling rate. The correct solution to the problem of tunneling of $\mathbf{M}$ in monodomain particles and to the problem of quantum nucleation of magnetic domains was given in Refs. 2 and 3.

${ }^{2}$ E. M. Chudnovsky and L. Gunther, Phys. Rev. Lett. 60, 661 (1988).

${ }^{3}$ E. M. Chudnovsky and L. Gunther, Phys. Rev. B 37, 9455 (1988).

${ }^{4}$ P. C. E. Stamp, Phys. Rev. Lett. 66, 2802 (1991); in Macroscopic Quantum Phenomena, edited by T. D. Clark et al. (World Scientific, Singapore, 1990).

${ }^{5}$ A. O. Caldeira and A. J. Leggett, Ann. Phys. (N.Y.) 149, 374 (1983); A. J. Leggett, Phys. Rev. B 30, 1208 (1984).

${ }^{6}$ T. Egami, Phys. Status Solidi A 20, 157 (1973); B 57, 211 (1973).

${ }^{7}$ B. Barbara et al., Solid State Commun. 10, 1149 (1972).

${ }^{8}$ O. Bostanjoglo and H. P. Gemund, Phys. Status Solidi A 17, 115 (1973); 48, 481 (1978).

${ }^{9}$ See, e.g., J. A. Baldwin and F. Milstein, J. Appl. Phys. 45, 4006
(1974); or W. Riehemann and E. Nemback, J. Appl. Phys. 55, 1081 (1983); 57, 476 (1985).

${ }^{10}$ M. Uehara and B. Barbara, J. Phys. (Paris) 47, 235 (1986).

${ }^{11}$ C. Paulsen et al., Phys. Lett. A 161, 319 (1991).

${ }^{12}$ X. X. Zhang et al., Phys. Lett. A 163, 130 (1992); J. Phys. C 4, L163 (1992).

${ }^{13}$ A. Garg and G. H. Kim, Phys. Rev. Lett. 63, 2512 (1989); Phys. Rev. B 43, 712 (1991).

${ }^{14}$ A. P. Malozemoff and J. C. Slonczewski, Magnetic Domain Walls in Bubble Materials (Academic, New York, 1979).

${ }^{15}$ J. S. Langer, Ann. Phys. (N.Y.) 41, 108 (1967).

${ }^{16} \mathrm{~S}$. Coleman, The Whys of Subnuclear Physics (Plenum, New York, 1979).

${ }^{17}$ The calcualtions of the dissipation for a planar wall (Ref. 4) are discussed in the review article of P. C. E. Stamp, E. M. Chudnovsky, and B. Barbara [Int. J. Mod. Phys. B 6, 1355 (1992)].

${ }^{18}$ See, e.g., A. J. Leggett and S. K. Yip, Chap. 8 in Helium Three, edited by W. Halperin and L. P. Pitaevskii (Elsevier, New York, 1990), Chap. 8.

${ }^{19}$ Y. Wada and J. R. Schrieffer, Phys. Rev. B 18, 3897 (1978).

${ }^{20}$ P. C. E. Stamp (unpublished). 